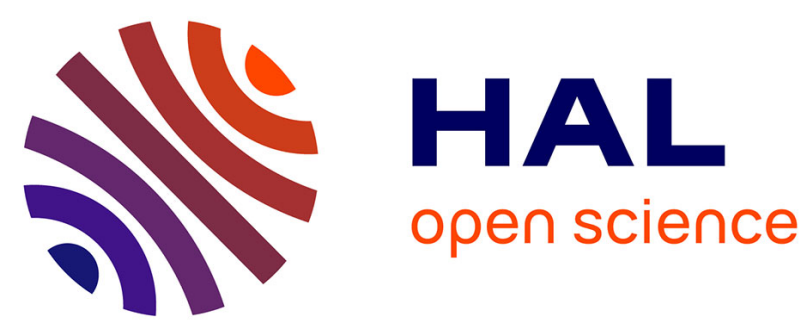

\title{
Birefringence measurements by means of light deflection at domain wal ls in ferroelastic crystals
}

P. Kolata, L. Guilbert, M.D. Fontana, Jean-Paul Salvestrini, Z. Czapla

\section{To cite this version:}

P. Kolata, L. Guilbert, M.D. Fontana, Jean-Paul Salvestrini, Z. Czapla. Birefringence measurements by means of light deflection at domain wal ls in ferroelastic crystals. Journal of the Optical Society of America B, 2000, 17 (12), pp.1973-1979. 10.1364/JOSAB.17.001973 . hal-00186030

\section{HAL Id: hal-00186030 \\ https://hal.science/hal-00186030}

Submitted on 2 Dec 2021

HAL is a multi-disciplinary open access archive for the deposit and dissemination of scientific research documents, whether they are published or not. The documents may come from teaching and research institutions in France or abroad, or from public or private research centers.
L'archive ouverte pluridisciplinaire HAL, est destinée au dépôt et à la diffusion de documents scientifiques de niveau recherche, publiés ou non, émanant des établissements d'enseignement et de recherche français ou étrangers, des laboratoires publics ou privés.

\section{(ㄷ)(1) $\$$}

Distributed under a Creative Commons Attribution - NonCommerciall 4.0 International 


\title{
Birefringence measurements by means of light deflection at domain walls in ferroelastic crystals
}

\author{
P. Kolata, L. Guilbert, M. D. Fontana, and J. P. Salvestrini \\ Laboratoire Matériaux Optiques Photonique et Systèmes, Université de Metz et Supelec, 2 rue Edouard Belin, \\ 57070 Metz, France \\ Z. Czapla \\ Institute of Experimental Physics, University of Wroclaw, Plac Maksa Borna 9, 50205 Wroclaw, Poland
}

\begin{abstract}
The deflection of light in ferroelastic crystals results from refraction and reflection at domain wa lls. When the tilt angle of the principal axes in neighboring domains is small, simple relationships between the crystal birefringences and the angles of the deflected beams can be deduced from Snell's law of $r$ efraction. As a rule, this condition is satisfied at $W$-domain $w$ alls in ferroelastic s pecies that have a biaxial prototype $p$ hase. In this case, measurement of the deflection a ngles permits o ne t o d etermine the b irefringences e asily. This method has as its main advantages independence of the sample thickness and the need for only rough sample preparation. It is absolutely insensitive to temperature fluctuations. We have applied the method to crystals of rubidium hydrogen selenate and dihydrated barium chloride as illustrative examples.
\end{abstract}

\section{INTRODUCTION}

In ferroelastic (FEL) crystals, the principal axes of permittivity are usually disoriented with respect to the domain walls. As a first consequence of this disorientation, the domain structure can be observed under a microscope between crossed polarizers. As another consequence, the optical discontinuity at the domain walls leads to the socalled deflection phenomenon: An incident light beam crossing the domain structure splits into several beams at the output of the crystal, as depicted in Fig. 1. This property of the crystal results from refraction and reflection at the domain walls. It was described by Tsukamoto et al. in for Rochelle salt, rubidium hydrogen selenate, gadolinium molybdate, and bismuth titanium oxide. ${ }^{1-5}$ The authors of those papers calculated deflection angles $\alpha$ and $\beta$ as functions of incidence angle $i$ by computing the Huygens-Fresnel principle at three interfaces (input face of the crystal, domain wall, and output face), assuming knowledge of all optical parameters of the crystal (values of the principal indices, orientation of the principal axes). By the way, the calculation dealing with Poynting vectors is not straightforward but is nevertheless exact in any case, regardless of the crystal symmetry.

Our aim in the present paper is to show that angles $\alpha$ and $\beta$ of the deflected beams can be directly related to birefringences-more precisely, to the differences $\Delta_{i}\left(n^{2}\right)$ - through simple analytical relationships deduced from Snell's law of refraction, provided that the tilt angle of the neutral lines with respect to the domain walls is small. This condition is actually fulfilled by the so-called $W$-domain walls (prominent reticular planes) in FEL crystals that belong to low-symmetry species, i.e., FEL species with a biaxial protophase. Thus the parameters $\Delta_{i}\left(n^{2}\right)$ of the FEL crystal can be deduced easily and accurately from angular measurements. Then the linear birefringences $\Delta n_{i}$ can be determined if one refractive index is known. The property of the method that differentiates it from conventional ones is that no special care is required in crystal preparation. Even knowledge of the sample thickness is not necessary. As in any method based on refractometry, temperature fluctuations during the experiments have negligible effects on the results compared with experimental errors. The method is applied here to two crystals at room temperature: rubidium hydrogen selenate $\left(\mathrm{RbHSeO}_{4}\right.$; $\left.\mathrm{RHSe}\right)$ and dihydrated barium chloride $\left(\mathrm{BaCl}_{2}: 2 \mathrm{H}_{2} \mathrm{O} ; \mathrm{BCD}\right)$.

\section{SYMMETRY CONSIDERATIONS: DEFINITION OF AXES}

Within our procedure we are interested in biaxial FEL species only, i.e., FEL species for which the prototype phase is either orthorhombic or monoclinic, and we consider the deflection of light at prominent reticular domain walls. With respect to these so-called $W$ walls (according to Sapriel's denomination ${ }^{6}$ ) the optical indicatrices in neighboring domains are inclined symmetrically. In biaxial FEL species the tilt angle is usually small, because this optical parameter is directly related to the FEL order parameter. It is thus possible to define a convenient system of three orthogonal axes $\left(x_{1}, x_{2}, x_{3}\right)$ to describe the optical properties of the multidomain crystal (Fig. 2):

- $x_{3}$, axis perpendicular to the domain walls;

- $x_{1}, x_{2}$, neutral lines in the plane of domain walls.

These so-called pseudoprincipal axes correspond to the average directions of the principal axes throughout the 


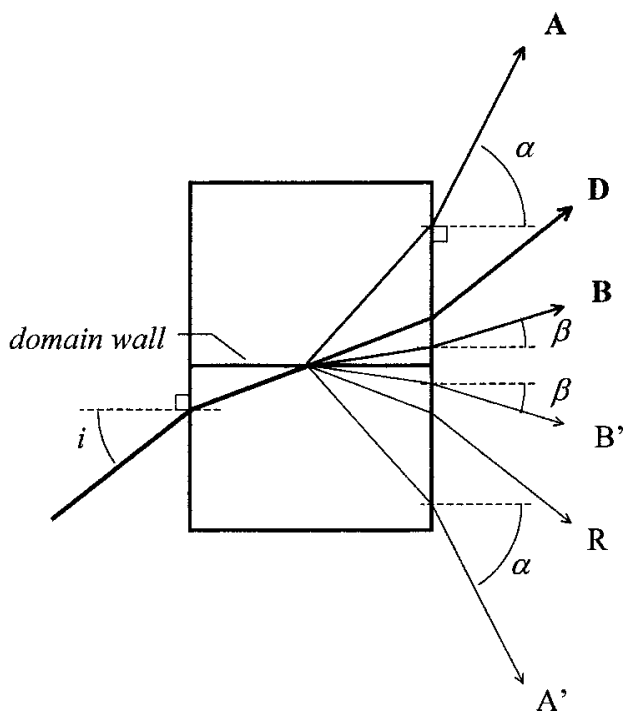

Fig. 1. Deflection phenomenon at a ferroelastic $W$-domain wall. A, B, refractive transmission (from low index to high index and vice versa), $\mathrm{A}^{\prime}$ and $\mathrm{B}^{\prime}$, the corresponding refractive reflections. $\mathrm{D}$ (direct beam) and $\mathrm{R}$ (reflected beam), quasi-nonrefractive processes without change of the index magnitude.
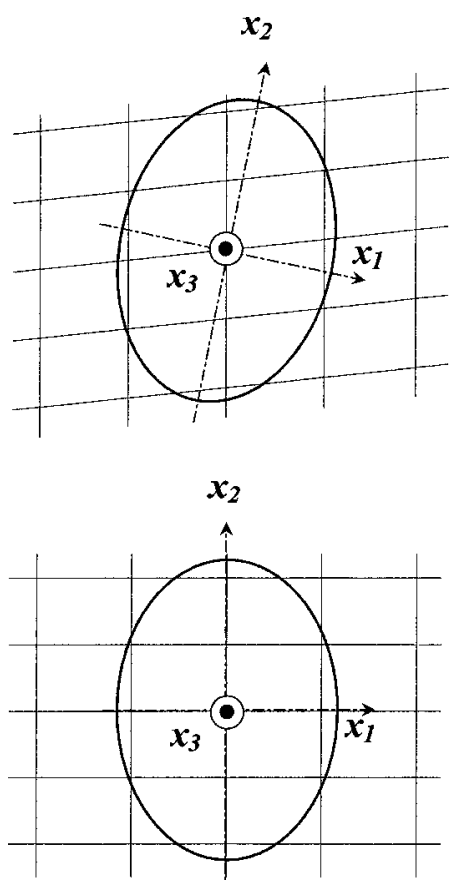

Fig. 2. Definition of the so-called pseudoprincipal axes: $x_{3}$, perpendicular to the plane of the domain walls; $x_{1}, x_{2}$, neutral lines in this plane. In the case of monoclinic crystals with orthorhombic protophase (bottom) $x_{1}$ and $x_{2}$ coincide with crystallographic axes. One of them is the monoclinic axis; it is a true principal axis that is common in both orientation states.

domain structure, and they should actually become the true principal axes in the prototype phase [i.e., above the Curie point, if a ferroelastic-paraelastic (FEL-PEL) phase transition actually exists]. Conventionally, $x_{1}$ and $x_{2}$ can be labeled such that the corresponding pseudoprincipal indices are ordered as $n_{1}<n_{2}$. In any domain of the FEL crystal, the orientation of the true principal axes relative to the pseudoprincipal axes can be fully described by angles $\pm \phi_{1}$ and $\pm \phi_{2}$ that are made by the neutral lines with respect to the $W$-domain walls in planes $\left(x_{2}, x_{3}\right)$ and $\left(x_{1}, x_{3}\right)$, respectively (Fig. 3$)$. In the lowestsymmetry case (triclinic FEL species), both tilt angles $\phi_{1}$ and $\phi_{2}$ are small but not zero. In monoclinic species with orthorhombic protophase, either $\phi_{1}$ or $\phi_{2}$ is strictly zero, because one of the axes (either $x_{2}$ or $x_{1}$ ) is the monoclinic axis and thus a true principal axis in both orientation states of the monoclinic structure.

In what follows, we shall suppose that the crystal sample is a plate cut perpendicularly to the $W$-walls-as is usually done in any deflection experiment by Tsukamoto et al. ${ }^{1-5}$ and as we do here. The plate will be designated by the cutting axis perpendicular to the surfaces: $x_{1}$ cut, $x_{2}$ cut, or any $x$ cut $[x$ axis lying in the plane $\left(x_{1}, x_{2}\right)$ of the domain walls].

The fact that the tilt angles are small in biaxial FEL species permits two important simplifications in the analytical treatment of the deflection phenomenon: (i) the three pseudoprincipal indices $n_{1}, n_{2}$, and $n_{3}$ are very close to the corresponding principal indices $n_{X}, n_{Y}$, and $n_{Z}$, so they can be merged into one another; (ii) in $x_{1}$ - and $x_{2}$-cut samples the polarization directions of all beams are never far from a principal axis or from a principal plane, except in the particular case when the beam propagates close to an optical axis of the biaxial crystal. So one of the beams, either the incident beam or the deflected beam, is quasi ordinary (that is, the corresponding refractive index is practically independent of the propagation direction). For the other beam the dependency of the extraordinary index follows a quasi-elliptical law as a func-
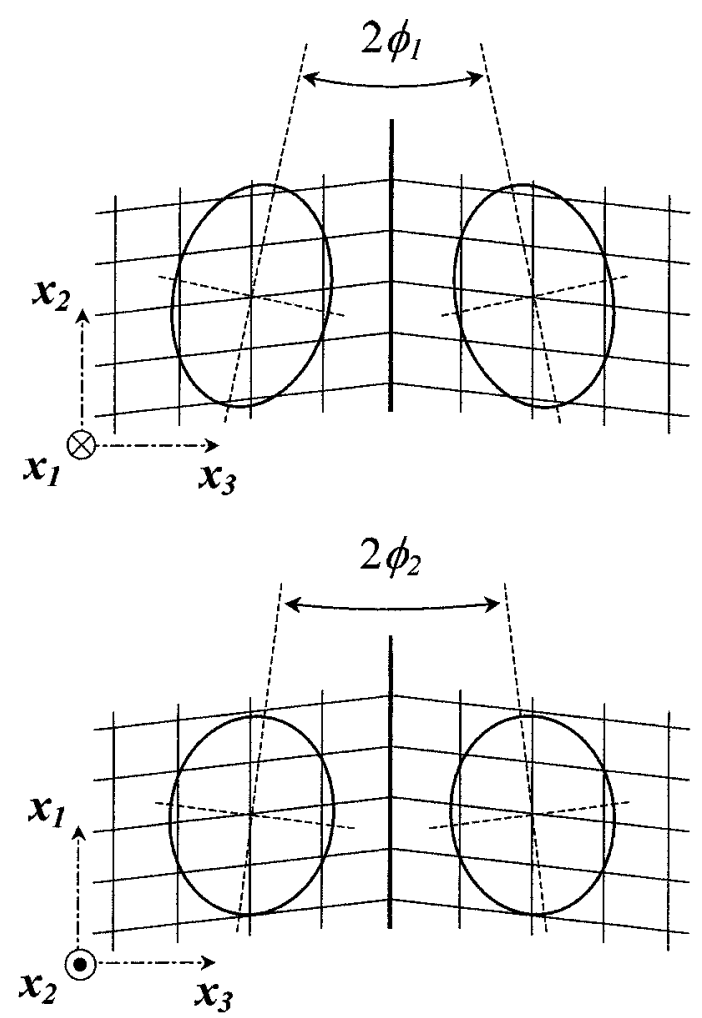

Fig. 3. Mutual tilt angle of the neutral lines with respect to a $W$-domain wall, as seen from pseudoprincipal axes $x_{1}$ and $x_{2}$ defined above (see Fig. 2). The figure shows the general case of a triclinic crystal. For monoclinic crystals either $\phi_{1}$ or $\phi_{2}$ is strictly zero. 
tion of the direction of the wave vector. The analytical calculations of the deflection angles that we shall develop below are based on these simplifications.

\section{RELATIONSHIPS BETWEEN DEFLECTION ANGLES AND BIREFRINGENCES}

When we consider the wave vectors instead of the Poynting vectors, Snell's law of refraction can be applied at the three interfaces: the input face, the domain wall, and the output face (Fig. 4). Inasmuch as the surfaces of the crystal are cut perpendicularly to the plane of the domain walls, the relations for the A deflection process (fast incident wave) and for the $\mathrm{B}$ deflection process (slow incident wave) are

A beam:

$$
\begin{gathered}
\sin i=n_{f}\left(i_{f}\right) \sin i_{f}, \quad n_{f}\left(i_{f}\right) \cos i_{f}=n_{s}\left(\alpha_{s}\right) \cos \alpha_{s}, \\
n_{s}\left(\alpha_{s}\right) \sin \alpha_{s}=\sin \alpha,
\end{gathered}
$$

B beam:

$$
\begin{gathered}
\sin i=n_{s}\left(i_{s}\right) \sin i_{s}, \quad n_{s}\left(i_{s}\right) \cos i_{s}=n_{f}\left(\beta_{f}\right) \cos \beta_{f}, \\
n_{f}\left(\beta_{f}\right) \sin \beta_{f}=\sin \beta,
\end{gathered}
$$

where $i$ is the external angle of incidence, $i_{f}\left(i_{s}\right)$ is the internal angle of the incident fast (slow) wave incident upon the domain wall, $n_{f}$ and $n_{s}$ are the corresponding low and high normal indices, $\alpha_{s}$ and $\beta_{f}$ are the internal angles of the deflected wave vectors after the domain wall (for the A and the B beams, respectively), and $\alpha$ and $\beta$ are the external deflection angles to be calculated. From Eqs. (1) and (2) separately, we obtain immediately and without approximation

$$
\begin{array}{ll}
\sin ^{2} \alpha-\sin ^{2} i=n_{s}{ }^{2}\left(\alpha_{s}\right)-n_{f}{ }^{2}\left(i_{f}\right) & (>0), \\
\sin ^{2} \beta-\sin ^{2} i=n_{f}{ }^{2}\left(\beta_{f}\right)-n_{s}{ }^{2}\left(i_{s}\right) & (<0) .
\end{array}
$$

Now, by introducing the simplifications that result from the small tilt angle of the principal axes with respect to the domain walls in biaxial FEL species (see Section 2), we can obtain approximate, but nevertheless correct, relations for deflection angles $\alpha$ and $\beta$ in both $x_{1}$-cut and $x_{2}$-cut samples. We shall first consider the case $n_{3}<n_{1}<n_{2}$, then $n_{1}<n_{2}<n_{3}$, and finally $n_{1}<n_{3}$ $<n_{2}$. (We have conventionally assumed that $n_{1}$ $\left.<n_{2}\right)$.

A. $n_{3}<n_{1}<n_{2}$

When $n_{3}<n_{1}<n_{2}$, the $\mathrm{A}$ beam is quasi ordinary in both $x_{1}$ - and $x_{2}$-cut samples (as well as in any $x$ cut). Thus the corresponding high index $n_{s}\left(\alpha_{s}\right)$ in Eq. (3) remains nearly equal to either $n_{2}$ ( $x_{1}$ cut) or $n_{1}$ ( $x_{2}$ cut), whatever the incidence is. The low-index $n_{f}\left(i_{f}\right)$, however, is extraordinary, but its variation is quasi elliptical to a good approximation:

$$
\frac{1}{n_{f}{ }^{2}\left(i_{f}\right)} \approx \frac{\cos ^{2} i_{f}}{n_{3}{ }^{2}}+\frac{\sin ^{2} i_{f}}{n_{j}{ }^{2}},
$$

where $n_{j}=n_{1}$ or $n_{2}$ (for $x_{1}$ - or $x_{2}$-cut samples, respectively). By combination of Eqs. (1) and approximation (5) we obtain, for $x_{1}$ - and $x_{2}$-cut samples, respectively,
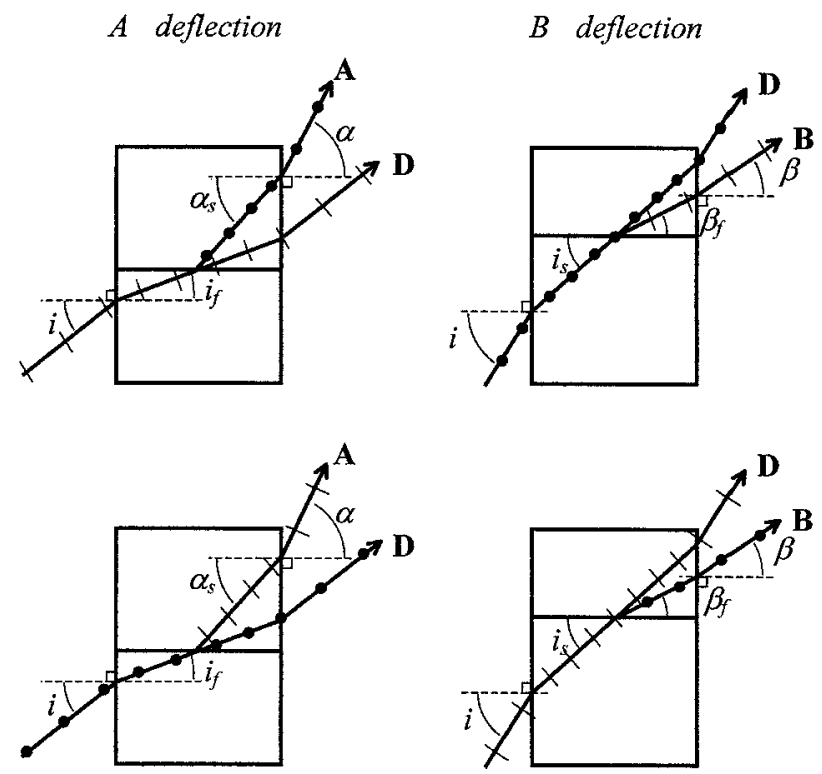

Fig. 4. Deflection processes, shown separately for the fast incident wave (A deflection) and for the slow incident wave (B deflection). For small optical tilts the beams are either quasi ordinary (filled circles) or extraordinary (hatch marks), depending on the refractive index (see Table 1) below. Deflections A and B are reciprocals of each other when the path of the light is reversed. The reflected $(R)$ and retrodeflected $\left(A^{\prime}\right.$ and $\left.B^{\prime}\right)$ beams, symmetrical to $\mathrm{D}, \mathrm{A}$, and $\mathrm{B}$, respectively, are not represented here.

$$
\begin{aligned}
& \sin ^{2} \alpha_{1}(i) \approx n_{2}{ }^{2}-n_{3}{ }^{2}+\frac{n_{3}{ }^{2}}{n_{1}{ }^{2}} \sin ^{2} i, \\
& \sin ^{2} \alpha_{2}(i) \approx n_{1}{ }^{2}-n_{3}{ }^{2}+\frac{n_{3}{ }^{2}}{n_{2}{ }^{2}} \sin ^{2} i .
\end{aligned}
$$

At zero incidence, angle $\alpha(0)$ of the A beams in any sample ( $x_{1}$ cut, $x_{2}$ cut, or any $x$ cut as well) is directly related to the birefringence of the crystal in the corresponding cutting plane:

$$
\begin{aligned}
& \sin ^{2} \alpha_{1}(0) \approx n_{2}{ }^{2}-{n_{3}}^{2} \equiv \Delta_{1}\left(n^{2}\right), \\
& \sin ^{2} \alpha_{2}(0) \approx n_{1}{ }^{2}-{n_{3}}^{2} \equiv \Delta_{2}\left(n^{2}\right), \\
& \sin ^{2} \alpha_{x}(0) \approx n^{2}(x)-n_{3}{ }^{2} .
\end{aligned}
$$

The quasi-ordinary index $n(x)$ in approximation (8) can be conveniently approximated by

$$
\frac{1}{n^{2}(x)} \approx \frac{\cos ^{2} x}{n_{2}{ }^{2}}+\frac{\sin ^{2} x}{n_{1}{ }^{2}}
$$

(angle $x$ designates the cutting axis with respect to the $x_{1}$ axis in the plane of domain walls.)

In the B deflection process [Eq. (4)] the incident slow wave is quasi ordinary (whatever the cutting axis $x$ and the incidence angle $i$ ), whereas the low index $n_{f}\left(\beta_{f}\right)$ of the deflected wave follows a quasi-elliptical law, similar to approximation (5). The $\mathrm{B}$ deflection process can be considered reciprocal to the A deflection process, by reversal of the light path. So one can deduce approximations (10) below from approximations (6) by changing $i$ into $\beta$ and $\alpha$ into $i$. Hence the following approximations for deflection angle $\beta$ of the B beam, in $x_{1}$ and $x_{2}$ cuts, respectively: 


$$
\begin{aligned}
& \frac{n_{3}^{2}}{n_{1}{ }^{2}} \sin ^{2} \beta_{1}(i) \approx \sin ^{2} i-\left(n_{2}{ }^{2}-n_{3}{ }^{2}\right), \\
& \frac{n_{3}^{2}}{n_{2}{ }^{2}} \sin ^{2} \beta_{2}(i) \approx \sin ^{2} i-\left(n_{1}{ }^{2}-n_{3}{ }^{2}\right) .
\end{aligned}
$$

B beams can appear only when angle of incidence $i$ exceeds a critical value, equal to deflection angle $\alpha(0)$ at zero incidence, given by approximations (7). This is of course a direct consequence of the $\mathrm{A}-\mathrm{B}$ reciprocity.

B. $n_{1}<n_{2}<n_{3}$ For $n_{1}<n_{2}<n_{3}$, the incident fast wave is quasi ordinary in $x_{1}$-cut as well as in $x_{2}$-cut plates (for small incidences). The corresponding low index $n_{f}\left(i_{f}\right)$ in Eq. (3) is nearly constant and equals either $n_{2}\left(x_{1}\right.$ cut) or $n_{1}\left(x_{2}\right.$ cut). The extraordinary index $n_{s}\left(\alpha_{s}\right)$ of the A beam follows a quasi-elliptical variation similar to that of approximation (5):

$$
\frac{1}{n_{s}{ }^{2}\left(\alpha_{s}\right)} \approx \frac{\cos ^{2} \alpha_{s}}{n_{3}{ }^{2}}+\frac{\sin ^{2} \alpha_{s}}{n_{j}{ }^{2}},
$$

where $n_{j}=n_{1}, n_{2}$ for $x_{1}$ or $x_{2}$ cut, respectively. By combining Eqs. (1) and approximation (11) we obtain, for $x_{1}$ and $x_{2}$ cuts, respectively,

$$
\begin{aligned}
& \frac{n_{3}{ }^{2}}{n_{1}{ }^{2}} \sin ^{2} \alpha_{1}(i) \approx n_{3}{ }^{2}-n_{2}{ }^{2}+\sin ^{2} i, \\
& \frac{n_{3}{ }^{2}}{n_{2}{ }^{2}} \sin ^{2} \alpha_{2}(i) \approx n_{3}{ }^{2}-n_{1}{ }^{2}+\sin ^{2} i .
\end{aligned}
$$

At zero incidence, these expressions lead to

$$
\begin{aligned}
& \sin ^{2} \alpha_{1}(0) \approx \frac{n_{1}{ }^{2}}{n_{3}{ }^{2}}\left(n_{3}{ }^{2}-n_{2}{ }^{2}\right)\left(x_{1} \text { cut }\right), \\
& \sin ^{2} \alpha_{2}(0) \approx \frac{n_{2}{ }^{2}}{n_{3}{ }^{2}}\left(n_{3}{ }^{2}-n_{1}{ }^{2}\right)\left(x_{2} \text { cut }\right) .
\end{aligned}
$$

Reciprocally, we can deduce the deflection angle $\beta(i)$ of the B beam (quasi ordinary) from relations (12) by reversing the path of light $(i \rightarrow \beta, \alpha \rightarrow i)$. Hence, for $x_{1}$ and $x_{2}$ cut, respectively,

$$
\begin{aligned}
& \sin ^{2} \beta_{1}(i) \approx \frac{n_{3}{ }^{2}}{{n_{1}}^{2}} \sin ^{2} i-\left(n_{3}{ }^{2}-n_{2}{ }^{2}\right), \\
& \sin ^{2} \beta_{2}(i) \approx \frac{n_{3}{ }^{2}}{{n_{2}}^{2}} \sin ^{2} i-\left(n_{3}{ }^{2}-n_{1}{ }^{2}\right) .
\end{aligned}
$$

C. $n_{1}<n_{3}<n_{2}$

When $n_{1}<n_{3}<n_{2}$, the $\mathrm{A}$ beam is quasi ordinary in $x_{1}$-cut plates, whereas the $\mathrm{B}$ beam is quasi ordinary in $x_{2}$-cut plates. Consequently, the deflection angle $\alpha(i)$ of the A beam follows relations (7) for $x_{1}$-cut and relations (12) for $x_{2}$-cut plates, whereas the deflection angle $\beta(i)$ of the $\mathrm{B}$ beam follows relation (10) for $x_{1}$-cut and relation (14) for $x_{2}$-cut plates.

\section{APPLICATION TO RUBIDIUM HYDROGEN SELENATE AND DIHYDRATED BARIUM CHLORIDE}

The structural and optical characteristics of rubidium hydrogen selenate (RHSe) are well known. ${ }^{7-9}$ At room temperature, RHSe belongs to the triclinic system. Its FEL domain structure consists of (001) $W$ walls that are related to $2 \mathrm{~F} 1 \mathrm{Aizu}$ species. Another family of $\mathrm{W}$ walls, parallel to (100) and related to $222 \mathrm{~F} 2$, is sometimes observed in this compound, but it was absent from our samples. $W^{\prime}$ walls are never observed. The tilt angles of the neutral lines in $x_{1}$ and $x_{2}$-cut plates are small, $\phi_{1}$ $=2.2^{\circ}$ and $\phi_{2}=0.6^{\circ}$, respectively. RHSe is thus a typical example of a biaxial FEL crystal that matches the required conditions for our simplified analytical treatment.

Dihydrated barium chloride (BCD) has monoclinic symmetry, with FEL $W$ walls parallel to (001) and related to an orthorhombic protophase. Because the $a$ axis is the monoclinic axis, tilt angle $\phi_{b}$ is strictly zero. The tilt angle measured in BCD $a$-cut plates between crossed polarizers $\left(\phi=7^{\circ}\right)$ is not so small as in RHSe.

Crystal plates of various orientations, typically $1 \mathrm{~mm}$ thick, are cut and polished from RHSe and BCD. Before cutting triclinic RHSe it is first necessary to place the asgrown crystal between crossed polarizers to find the neutral lines $x_{1}$ and $x_{2}$ in plane (001) of the domain walls. In BCD these two axes are easy to find because they coincide with orthogonal axes $a$ and $b$ of the monoclinic structure. However, according to our convention introduced in Section $3\left(n_{1}<n_{2}\right)$, the proper assignment of $x_{1}$ and $x_{2}$ to either $a$ or $b$ in monoclinic crystals can be made only after deflection experiments. That is why we shall now refer to BCD plates as either $a$ cut or $b$ cut.

Measurements of the deflection angles are performed with a goniometer with a typical error of $\pm 0.1^{\circ}$. Both the incident laser beam $(633 \mathrm{~nm})$ and the crystal plate are carefully centered on the rotation axis of the setup. The polarization of the incident beam is set linear by a polarizer to extinguish either B deflection or A deflection. An analyzer is also used to check the polarization of the deflected beams. In this way it is easy to determine which one of the deflected beams is quasi ordinary and thus to sort the pseudoprincipal indices among the three possible cases examined in Section 3 and listed in Table 1. For a better accuracy, each angular measurement is repeated after the sample is rotated by $180^{\circ}$ to bring to light-and eventually suppress by averaging - any possible error owing to an off-centering of the sample with respect to the rotation axis. The systematic error that is due to finite sample thickness is estimated in Appendix A.

It should be noted that FEL domain structure usually yields not only deflection but also diffraction. However, because domain walls in as-grown crystals are randomly distributed, this diffraction consists of broad lines surrounding the deflection spots, whatever the number of domain walls is. So, most often diffracted light is not 
Table 1. Characteristics of the Deflected Beams at Small Incidence ${ }^{a}$

\begin{tabular}{clcc}
\hline \multirow{2}{*}{$\begin{array}{c}\text { Crystal } \\
\text { Cut }\end{array}$} & \multicolumn{3}{c}{ Indices } \\
\cline { 2 - 4 } & $n_{1}<n_{2}<n_{3}$ & $n_{3}<n_{1}<n_{2}$ & $n_{1}<n_{3}<n_{2}$ \\
\hline$x_{1}$ & A beam extraordinary, & A beam quasi ordinary, & A beam quasi ordinary, \\
& B beam quasi ordinary & B beam extraordinary & B beam extraordinary \\
$x_{2}$ & A beam extraordinary, & A beam quasi ordinary, & A beam extraordinary, \\
& B beam quasi ordinary & B beam extraordinary & B beam quasi ordinary \\
\hline
\end{tabular}

${ }^{a}$ Depending on the sort of refractive index (by convention, $x_{3}$ is the axis perpendicular to the plane of the $W$-domain walls).

troublesome: Deflection spots are easy to recognize and to localize because they are much brighter.

Complete sets of measurements versus angle of incidence have been recorded in both $x_{1}$-cut and $x_{2}$-cut RHSe samples. Inasmuch as the A beam is quasi ordinary in both plates (A polarization nearly perpendicular to the plane of incidence) we can conclude that RHSe matches the first case that we examined above $\left(n_{3}<n_{1}<n_{2}\right)$. The experimental results $\alpha(i)$ and $\beta(i)$ at $633 \mathrm{~nm}$ are shown in Fig. 5, together with the theoretical fits calculated from approximations (6) and (10). Linear regressions of $\sin ^{2} \alpha$ and $\sin ^{2} \beta$ versus $\sin ^{2} i$ yield the following fitting parameters:

$$
\begin{aligned}
& x_{1} \text { cut: } \\
& \qquad \begin{array}{c}
\Delta_{1}\left(n^{2}\right) \equiv n_{2}{ }^{2}-n_{3}{ }^{2}=0.1716(12), \\
n_{3}{ }^{2} / n_{1}{ }^{2}=0.984(4),
\end{array}
\end{aligned}
$$

$x_{2}$ cut:

$$
\begin{gathered}
\Delta_{2}\left(n^{2}\right) \equiv n_{1}{ }^{2}-n_{3}{ }^{2}=0.0420(6), \\
n_{3}{ }^{2} / n_{2}{ }^{2}=0.930(2) .
\end{gathered}
$$

The most pertinent parameters in these fittings are the values of $\Delta_{i}\left(n^{2}\right)$. For this purpose single measurements at zero incidence are sufficient. Then the linear birefringences $\Delta n_{i}$ can be deduced if one index is known. If it is not, one can, in principle, use the least-squares values of the index ratios $n_{3} / n_{j}$ that fit the deflection data in the full angular range to determine the three refractive indices. But this method is inaccurate unless the deflection measurements are done with a very high angular accuracy $(<0.01$ error; see below, Appendix A). For preferance we used the value of $n_{2}$ previously measured in RHSe at $633 \mathrm{~nm}$ by interferometry ${ }^{9}\left(n_{2}=1.5632\right)$ to yield the linear birefringences from our deflection data at zero incidence:

$$
\begin{aligned}
& \Delta n_{1} \approx n_{2}-n_{3}=0.0559(4), \\
& \Delta n_{2} \approx n_{3}-n_{1}=-0.0139(2) .
\end{aligned}
$$

For BCD crystal, complete measurements of deflection angles $\alpha(i)$ and $\beta(i)$ are made for the $a$-cut plate only [Fig. 6(a)]. In principle, $b$-cut plates cause no deflection, owing to the monoclinic symmetry. Nevertheless, it is possible to tilt the $b$-cut plate slightly by a few degrees about the axis perpendicular to the domain walls such that a weak deflection reappears. In this way, deflection
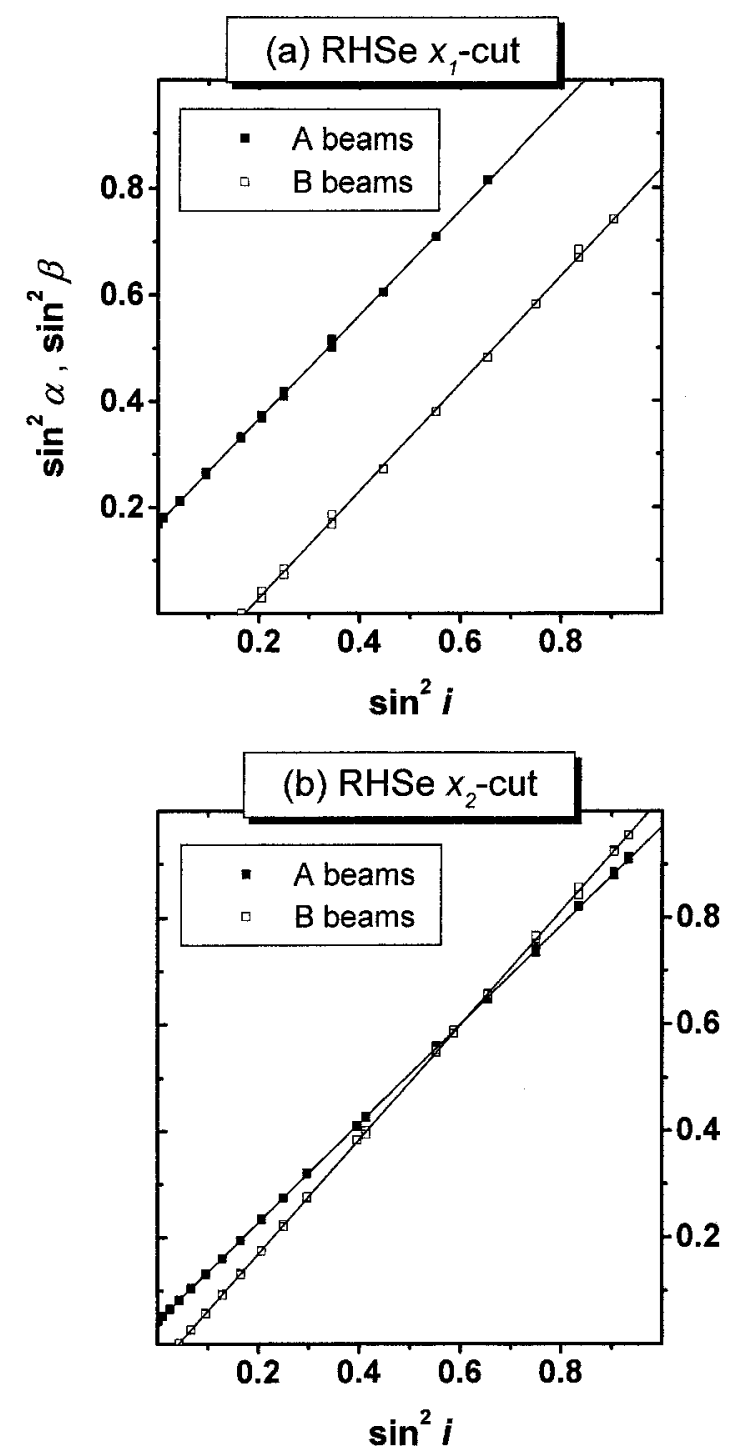

Fig. 5. Deflection angles measured in $x_{1}$-cut and $x_{2}$-cut RHSe plates. The solid curves are linear regressions. In the experiment, $x_{2}$-cut beam crossing occurs when the incident wave vector comes close to an optical axis.

angle $\alpha(0)$ at zero incidence can be measured in the vicinity of the $b$ axis and then interpolated by a parabolic fitting [Fig. 6(b)]. From polarization considerations it can be concluded that the index sequence in BCD is $n_{b}<n_{a}$ $<n_{3}$ (so $x_{1} \equiv b$ axis). Using approximations (13), we can deduce the birefringences of BCD at $633 \mathrm{~nm}$ from the deflection angles at zero incidence: 
in $a$ cut plates,

$$
\begin{aligned}
\alpha_{2}(0) & =17.2( \pm 0.1)^{\circ} \\
& \Rightarrow \Delta_{a}\left(n^{2}\right) \\
& \equiv n_{3}{ }^{2}-n_{b}{ }^{2} \\
& \approx\left(n_{3}{ }^{2} / n_{a}{ }^{2}\right) \sin ^{2} \alpha_{2}(0) \\
& =0.0888(10),
\end{aligned}
$$

in $b$ cut plates,

$$
\begin{aligned}
\alpha_{1}(0) & =11.4( \pm 0.1)^{\circ} \\
& \Rightarrow \Delta_{b}\left(n^{2}\right) \\
& \equiv n_{3}{ }^{2}-n_{a}{ }^{2} \\
& \approx\left(n_{3}{ }^{2} / n_{b}{ }^{2}\right) \sin ^{2} \alpha_{1}(0) \\
& =0.0404(8) .
\end{aligned}
$$
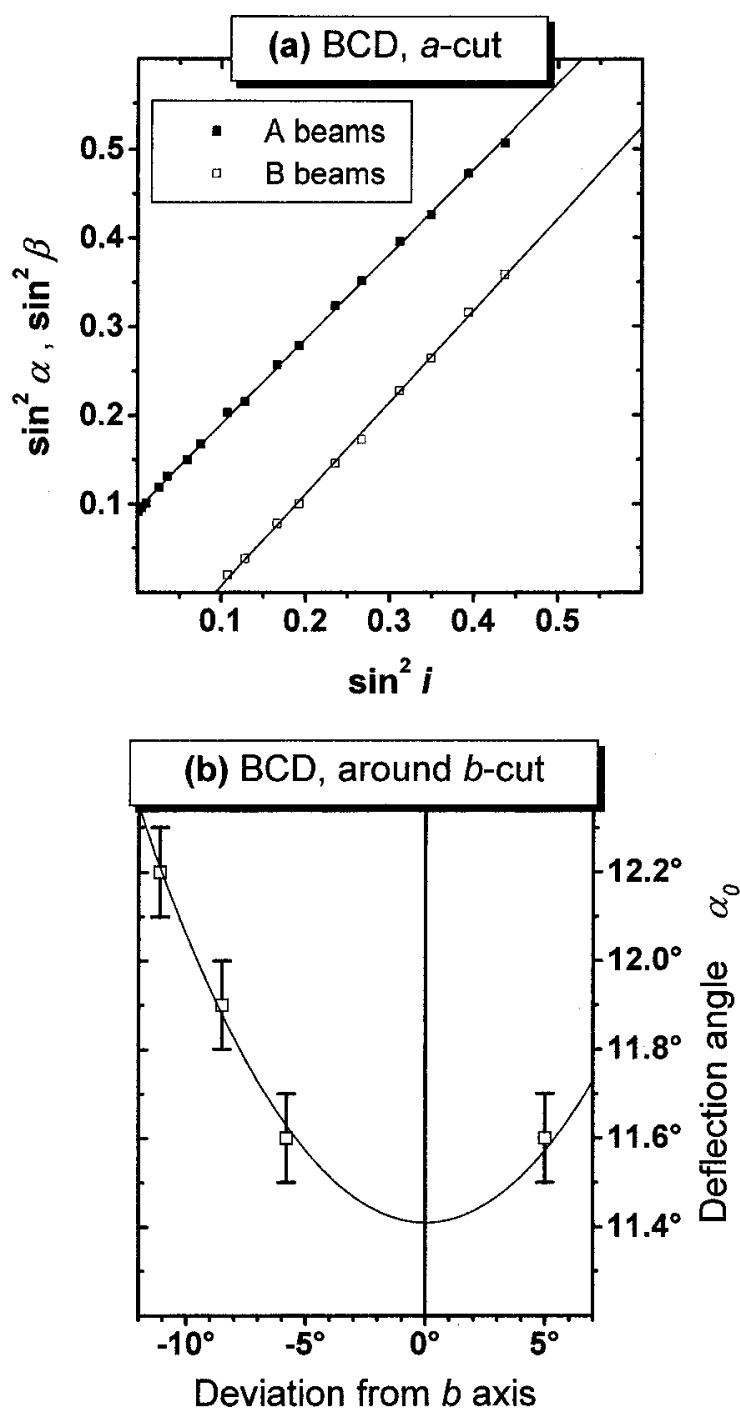

Fig. 6. Deflection angles measured in BCD plates. The solid curves in (a) are linear regressions. The $b$-cut plate (b) causes no deflection, owing to its monoclinic symmetry; deflection angle $\alpha_{b}(0)=11.4^{\circ}$ is determined by a parabolic interpolation of angular measurements in the vicinity of the $b$ axis.
If an exact computation method similar to that of Tsukamoto et al., is used, the corresponding values of the birefringences that match the experimental results should differ from the values given above by less than $2 \times 10^{-4}$. So it can be concluded that our approximate treatment is still fully valid for such crystals as $B C D$ for which the optical tilt angle is $\sim 7^{\circ}$. Except for strongly birefringent crystals, the relative error on $\Delta\left(n^{2}\right)$ stays below $0.5 \%$ when the optical tilt angle does not exceed $8^{\circ}$.

\section{CONCLUSIONS}

The phenomenon of light deflection that occurs at $W$-domain walls in ferroelastic crystals can be used to determine birefringence. For monochromatic experiments the setup consists simply of a goniometer and a laser source. For measurements at several wavelengths it is of course possible to replace the laser source with a spectral lamp or with a white source associated with a monochromator. The method does not require big crystal samples (they can be as small as $2 \mathrm{~mm} \times 2 \mathrm{~mm} \times 1 \mathrm{~mm}$ ), provided that they are cut perpendicular to the domain walls. Unlike for conventional methods, the quality of the sample is not critical for accuracy, and knowledge of the sample's thickness is not necessary. Moreover, simple considerations of the polarizations of the deflected beams permit one to sort the refractive indices easily. As has been shown here, the use of ferroelastic species related to a biaxial protophase is especially favorable for this purpose because the small optical tilt at domain walls leads to simple analytical relationships between the deflection angles and the birefringences. Except for strongly birefringent crystals, these approximate relationships remain valid for tilt angles as large as $8^{\circ}$, within a relative error of $<0.5 \%$ on $\Delta\left(n^{2}\right)$. For larger tilt angles (twinned crystals or FEL crystals with uniaxial protophase) a refined analytical treatment is required for determining moreaccurate relationships between the deflection angles and the birefringences. ${ }^{10}$

\section{APPENDIX A: SYSTEMATIC ERROR OWING TO SAMPLE THICKNESS}

For measurements of the deflection angles we prefer to use a narrow beam (usually a laser beam) focused upon the crystal or upon a diaphragm mounted upon the rotating arm of a goniometer. For large crystal plates $(>1$ $\mathrm{cm}^{2}$ ) it is possible to use a wide beam in a classical goniometer with input and output collimators. But for small samples the first method is the more convenient one. In this case it is necessary to center the sample carefully upon the rotation axis of the goniometer. We achieve centering by ensuring that the same value of the deflection angle is measured when the sample has been rotated by $180^{\circ}$. Nevertheless, a systematic error remains, which is due to the sample thickness. This error always occurs by default. It is of course proportional to the ratio $t / L$ ( $t$ is the sample thickness; $L$ is the length of the arm of the goniometer). From simple trigonometric considerations (Fig. 7) we obtain immediately (for small incidences) 


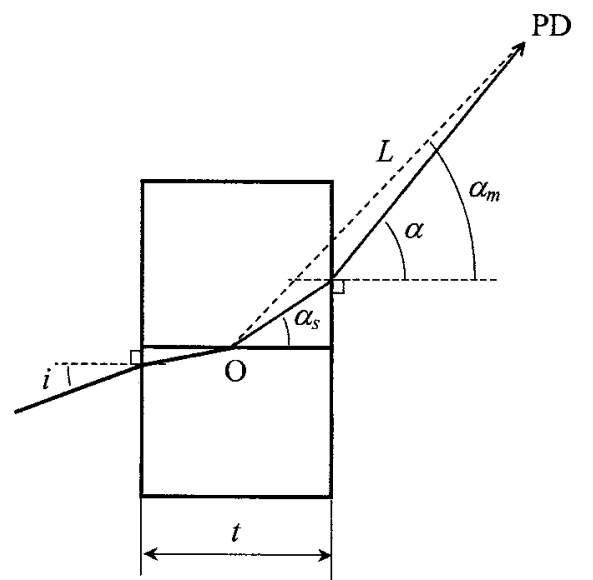

Fig. 7. Systematic error caused by sample thickness. PD, photodetector.

$$
\begin{aligned}
\delta \alpha & \equiv \alpha-\alpha_{m}=\frac{t \cos ^{3} \alpha}{2 L}\left(\tan \alpha-\tan \alpha_{s}\right) \\
& \approx \frac{t}{2 L}(1-1 / n) \sin \alpha
\end{aligned}
$$

where $\alpha$ is the deflection angle (exact), $\alpha_{m}$ is the measured value, $\alpha_{s}$ is the internal angle, and $n$ is the refractive index.

In our experiments, sample thickness $t$ was $\sim 1 \mathrm{~mm}$ and arm length was $L=200 \mathrm{~mm}$. These values yield small systematic error of $\sim 0.02^{\circ}$ for the deflection angle. However, for large angles of incidence this systematic error arises slowly, as do other errors that could result from several factors (broadening of the deflected beams, bad alignment of the sample surfaces). For this reason the general accuracy of the method cannot usually be better than $0.05^{\circ}$ unless the surfaces are perfectly polished and parallel and the systematic error is conveniently cor- rected. That is why we do not recommend using the measurements made at large angle to fit the index ratios, as could be tentatively done, for instance, in Eqs. (15) and (16). The present method is suitable, as a rule, for determining only the birefringences, not the refractive indices.

L. Guilbert's e-mail address is guilbert@ese-metz.fr.

\section{REFERENCES}

1. T. Tsukamoto, J. Hatano, and H. Futuma, in "Proceedings of the 2nd Japanese-Soviet Symposium on Ferroelectricity, Kyoto 1980," J. Phys. Soc. Jpn. 49, Suppl. B 155-157 (1980).

2. T. Tsukamoto, M. Komuake, S. Suzuki, H. Futuma, and Y. Makita, "Domain structure and deflection of light at domain walls in $\mathrm{RbHSeO}_{4}$," J. Phys. Soc. Jpn. 52, 3966-3973 (1983).

3. T. Tsukamoto, J. Hatano, and H. Futuma, "Refraction and reflection of light at ferroelastic domain walls in rochelle salt crystal,” J. Phys. Soc. Jpn. 51, 3948-3952 (1982).

4. T. Tsukamoto, J. Hatano, and H. Futuma, "Deflection of light by ferroelastic domains in $\mathrm{Gd}_{2}\left(\mathrm{MoO}_{4}\right)_{3}$ and $\mathrm{BiTi}_{3} \mathrm{O}_{12}$ crystals," J. Phys. Soc. Jpn. 53, 838-843 (1984).

5. T. Tsukamoto and H. Futuma, "Light deflection induced by ferroelastic layered domains," Phase Transit. 45, 59-76 (1993).

6. J. Sapriel, "Domain-wall orientations in ferroelastics," Phys. Rev. B 12, 5128-55140 (1975).

7. R. Poprawski, J. Mroz, Z. Czapla, and L. Sobczyk, "Ferroelectrics properties and domain structure in $\mathrm{RbHSeO}_{4}$," Acta Phys. Pol. A 55, 641-645 (1979).

8. I. Brach, D. J. Jones, and J. Roziére, "The crystal structure of $\mathrm{RbHSeO}_{4}$ : a neutron diffraction study of the paraelectric phase," J. Solid State Chem. 48, 401-406 (1983).

9. L. Guilbert, J. P. Salvestrini, P. Kolata, F. X. Abrial, M. D. Fontana, and Z. Czapla, "Optical characteristics of triclinic rubidium hydrogen selenate,” J. Opt. Soc. Am. B 15, 10091016 (1998).

10. J. Bornarel, P. Staniorowski, and Z. Czapla, "Light deflection and birefringence in APFA ferroelastic crystals," J. Phys.: Condens. Matter 12, 653-667 (2000). 